\title{
Identification of a compound heterozygote in LYST gene: a case report on Chediak- Higashi syndrome
}

\author{
Yinsen Song ${ }^{1 \dagger}$, Zhengping Dong ${ }^{2 \dagger}$, Shuying Luo ${ }^{3}$, Junmei Yang ${ }^{1}$, Yuebing $\mathrm{Lu}^{3}$, Bo Gao ${ }^{4^{*}}$ and Tianli Fan ${ }^{5^{*}}$ (D
}

\begin{abstract}
Background: Chediak-Higashi Syndrome (CHS) is a rare autosomal recessive disease caused by loss of function of the lysosomal trafficking regulator protein. The causative gene LYST/CHS1 was cloned and identified in 1996, which showed significant homology to other species such as bovine and mouse. To date, 74 pathogenic or likely pathogenic mutations had been reported.

Case presentation: Here we describe a compound heterozygote in LYST gene, which was identified in a 4-year-old female patient. The patient showed skin hypopigmentation, sensitivity to light, mild splenomegaly and reduction of platelets in clinical examination. Giant intracytoplasmic inclusions were observed in the bone marrow examination, suggesting the diagnosis of CHS. Amplicon sequencing was performed to detect pathogenic mutation in LYST gene. The result was confirmed by two-generation pedigree analysis base on sanger sequencing.

Conclusion: A compound heterozygote in LYST gene, consisting of a missense mutation c.5719A > G and an intron mutation c.4863-4G > A, was identified from the patient by using amplicon sequencing. The missense mutation is reported for the first time. Two-generation pedigree analysis showed these two mutations were inherited from the patient's parents, respectively. Our result demonstrated that amplicon sequencing has great potential for accelerating and improving the diagnosis of rare genetic diseases.
\end{abstract}

Keywords: Chediak-Higashi syndrome, LYST gene, Compound heterozygote, Amplicon sequencing, Two-generation pedigree

\section{Background}

Chediak-Higashi Syndrome (CHS, MIM: 214500) rare but lethal autosomal recessive disorder characterized by multiple clinical features, including hypopigmentation of skin and hair, reduction of platelets and leukocytes, abnormal organelles in circulating granulated cells, and neurological dysfunction [1, 2]. Recurrent infections could also be observed due to severe immunodeficiency. The primary immunologic symptom lymphoproliferative histiocytosis, which called the accelerated phase determines the prognosis of CHS [3]. Patients with early-onset accelerated phase

\footnotetext{
* Correspondence: fromzero1121@hotmail.com; tlfan2012@163.com ${ }^{\dagger}$ Yinsen Song and Zhengping Dong contributed equally to this work. ${ }^{4}$ Department of Laboratory Medicine, Taihe Hopsital, Hubei University of Medicine, Shiyan, China

${ }^{5}$ School of Basic Medical Sciences, Zhengzhou University, Zhengzhou 450001, Henan, China

Full list of author information is available at the end of the article
}

(before the age of 6) often have severe phenotype and died in the first decade, however, about $10-15 \%$ of patients with the late-onset forms follow a less severe clinical course of $\mathrm{CHS}$ and survive without having treatment of bone marrow transplantation $[4,5]$.

CHS was first described in 1950s [6], but the causative gene had not been revealed until 1996 [7]. The LYST gene (HGNC:1968), consisting of 53 exons with a mRNA transcript of $13,503 \mathrm{bp}$, was identified to be responsible for this disease $[8,9]$. Previous studies suggested that the product of the LYST gene is required for sorting endosomal resident proteins into late multivesicular endosomes [10]. To date, 74 pathogenic or likely pathogenic mutations in LYST gene had been reported (Additional file 2: Table S1), correlations between genotypes and phenotypes had been investigated.

Here we describe a compound heterozygote in LYST gene, consisting of a missense mutation NM_000081.2:

(c) The Author(s). 2019 Open Access This article is distributed under the terms of the Creative Commons Attribution 4.0 International License (http://creativecommons.org/licenses/by/4.0/), which permits unrestricted use, distribution, and 
c.5719A > G (p.Ile1907Val) and a intron mutation $\mathrm{NC}_{-}$ 000001.10:g.235945391C > T (c.4863-4G > A), which was identified in a 4-year-old female patient with $\mathrm{CHS}$. $\mathrm{Mu}$ tations in LYST gene were detected by using amplicon sequencing within $24 \mathrm{~h}$. We also performed sanger sequencing to confirm the result and identify the source of mutations in the patient's family. A typical autosomal recessive inheritance pattern was demonstrated by our data. Our finding expands the spectrum of pathogenic mutations in LYST gene and indicates an efficient and accurate approach for diagnosis of genetic diseases.

\section{Case presentation}

The 4-year-old female patient was admitted to Children's Hospital Affiliated to Zhengzhou University with recurrent upper respiratory infections last for 2 months. Antibiotic treatment had been taken but without any improvement. Patient and her parents are all Han Chinese from Henan province of China, no family history was noticed in records. The study was approved by the ethics committee of Children's Hospital Affiliated to Zhengzhou University. A written informed consent for publishing data and images was obtained from the parents before performing genetic test.

The patient had low fever and cough, temperature was measured to $38.5^{\circ} \mathrm{C}$ on regular examination. Hypopigmentation of skin on neck and knee was observed on physical examination. Slight hepatomegaly was detected by supersonic inspection. Blood analysis showed reduction of platelets (PLT, $88^{*} 10^{9} / \mathrm{L}$ ) and white blood cell (WBC, $2.7^{*} 10^{9} / \mathrm{L}$ ), the red blood cell was normal (RBC, $\left.4.9^{*} 10^{12} / \mathrm{L}\right)$. Leukocytes with giant intracytoplasmic inclusions were observed with peripheral smear, which evidenced by bone marrow aspirate (Fig. 1). The neurological examination was normal. Clinical manifestations suggested the diagnosis of CHS. Genetic test for the patient and her parents was recommended.

\section{Identification of causative mutations in LYST gene}

A customized panel covering all 53 exons and $10 \mathrm{bp}$ of padding region of LYST gene was designed by using the online tool (https://www.ampliseq.com), details was described in Additional file 2: Table S1. Genomic DNA was purified from peripheral blood mononuclear cells (PBMC) with widely used commercial kit (Tiangen Biotech, Beijing, China) according to manufacturer's protocol. DNA library was prepared by multiple PCR in one tube and sequenced with the Ion S5XL genetic analyzer (ThermoFisher Scientific, Waltham, MA, USA). Mutations were detected by using VariantCaller V1.0, along with human genome reference hg19 (GRCh37). Clinical significance of mutations was predicted by using SIFT (Version 5.11) [11] and Human Splicing Finder (Version 3.1) [12].

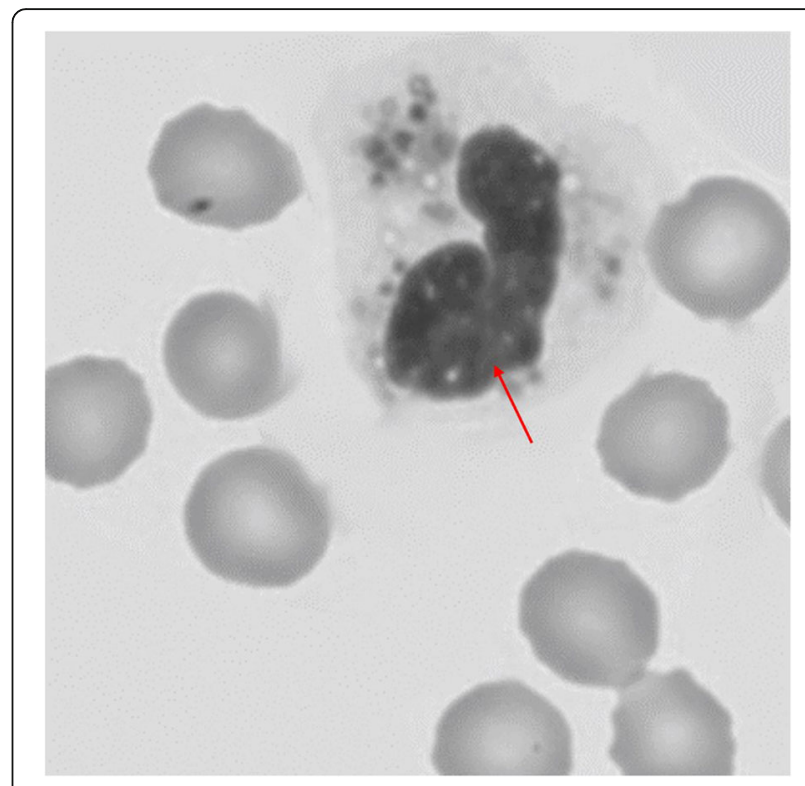

Fig. 1 Bone marrow examination for patient with CHS. Giant intracytoplasmic inclusion in leukocyte is indicated by red arrow

As result, two heterozygous mutations were detected, including one missense mutation NM_000081.2: c.5719A > G (p.Ile1907Val) and a intron mutation NC_ 000001.10:g.235945391C > T (c.4863-4G > A) (Table 1). The intron mutation c.4863-4G $>\mathrm{A}$ is a known SNP (rs201382097) which distributes mainly in Asian (Additional file 3: Table S2 and Additional file 4: Table S3), our data indicates likely pathogenic by affect splicing (Additional file 1). The missense mutation c.5719A > G locates in exon19 of LYST gene, resulting an amino acid sequence change of I1907V, is reported for the first time and predicted to be damaging for protein function by SIFT. The result was confirmed by sanger sequencing. Two-generation pedigree analysis was performed, targeting these two mutations. A typical autosomal recessive inheritance pattern was demonstrated, the missense mutation c.5719A > G was inherited from patient's mother, the other was from father (Fig. 2).

To further confirm the influence caused by these two mutations, an ELISA test for LYST protein was performed using commercial ELISA kit purchased from Cusabio Bioengineering co. LTD (Wuhan, China). $400 \mu \mathrm{L}$ of plasma from the patient and her father was used to extract protein, then tested according to manufacturer's protocol. The result showed that normal LYST protein is undetectable in plasma of the patient, proving the loss of function of LYST protein (Fig. 3).

\section{Discussion and conclusions}

Here we described a compound heterozygote in LYST gene identified from a 4-year-old female patient in China, who was diagnosed with CHS based on clinical 
Table 1 Identified variations from the patient with CHS

\begin{tabular}{|c|c|c|c|c|c|c|}
\hline Variation & Location & Peptide change & Type & Zygosity & ID in dbSNP & Allele Frequency in gnomAD \\
\hline NM_000081.2: c.5719A > G & Chr1:235937207 & p.lle1907Val & missense & Heterozygous & not applicable & not applicable \\
\hline NC_000001.10: g.235945391C> T & Chr1: 235945391 & $\mathrm{p} .=$ & intron & Heterozygous & rs201382097 & 0.0006894 \\
\hline NM_003664.3: c.1754 T > A & Chr5:77425028 & p.Val585Glu & missense & Heterozygous & rs6453373 & 0.8668 \\
\hline NM_001083116.1: c.900C > T & Chr10:72358577 & $\mathrm{p} .=$ & synonymous & Heterozygous & rs885822 & 0.6403 \\
\hline NM_199242.2: c.3198A > G & Chr17:73824121 & $\mathrm{p} .=$ & synonymous & Heterozygous & rs7210574 & 0.3966 \\
\hline NM_199242.2: c.2599A > G & Chr17:73827205 & p.Lys867Glu & missense & Heterozygous & rs1135688 & 0.3626 \\
\hline NM_199242.2: c.1977C > T & Chr17:73831016 & $\mathrm{p} .=$ & synonymous & Heterozygous & rs2290770 & 0.02977 \\
\hline NM_199242.2: c.888G >C & Chr17:73836162 & $\mathrm{p} .=$ & synonymous & Heterozygous & rs3744026 & 0.1496 \\
\hline NM_199242.2: c.279C > T & Chr17:73839137 & $\mathrm{p} .=$ & synonymous & Heterozygous & rs3744007 & 0.04832 \\
\hline NM_006949.2: c.38-7C > T & Chr19:7703605 & $\mathrm{p} .=$ & intron & Heterozygous & rs8104339 & 0.4047 \\
\hline NM_006949.2: c.1443 T > C & Chr19:7711221 & $\mathrm{p} .=$ & synonymous & Heterozygous & rs10001 & 0.4204 \\
\hline NM_006949.2: c.1576A > G & Chr19:7712277 & p.lle526Val & missense & Heterozygous & rs6791 & 0.6401 \\
\hline NM_001204401.1: c.1268A >C & ChrX:123034511 & p.Gln423Pro & missense & Heterozygous & rs5956583 & 0.3378 \\
\hline
\end{tabular}

manifestations including hypopigmentation of skin, hepatomegaly, reduction of PLT and WBC, and leukocytes with giant intracytoplasmic inclusions. Genetic test showed two heterozygous mutations were inherited from her father and mother, respectively. The girl was treated with enhanced anti-infective therapy and liver protection, the clinical manifestation was stabilized. Further treatment including HSCT will be evaluated based on conditions of prognosis.

To date, 74 pathogenic or likely pathogenic mutations in LYST gene had been reported in ClinVar database (https://www.ncbi.nlm.nih.gov/clinvar/) (Additional file 5:
Table S4), the genotype-phenotype correlations had been investigated in the past two decades [9]. In general, lossof-function variants such as nonsense or frame-shift mutations are associated with severe, childhood-onset form and missense variants with milder adolescent- or adultonset forms of the disorder $[4,13]$. In this case, a compound heterozygote consisting of a missense and an intron mutation was identified. The intron mutation c.4863-4G > A was predicted to be probably affecting splicing, might lead to loss-of-function and cause severe conditions. In this case, phenotype of the patient was mild and stabilized for now, but long-term nursing is

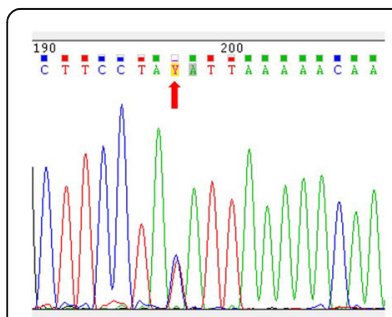

Father: LYST:c.4863-4G>A
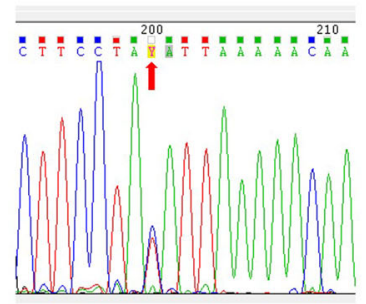

Patient: LYST:c.4863-4G>A

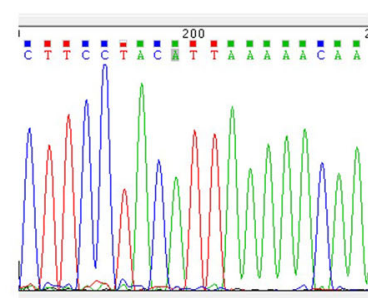

Mother: no mutation

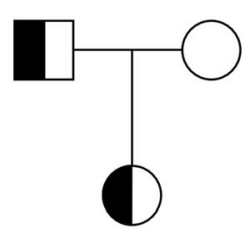

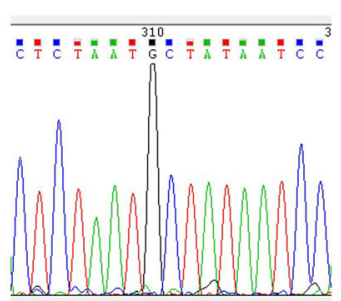

Father: no mutation

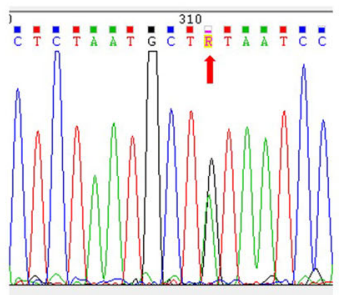

Patient: LYST:c.5719A >G

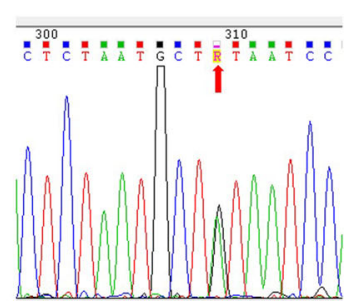

Mother: LYST:c.5719A >G

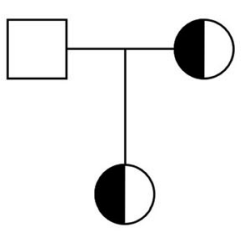

b

Fig. 2 Identified variations in LYST gene. a. Reported intron mutation (c.4863-4G > A) in exon15, inherited from father. b. Novel missense mutation $(c .5719 \mathrm{~A}>\mathrm{G})$ in exon19, inherited from mother. Mutations are marked with red arrow. Zygosity is showed by letters of degenerate bases ( $\mathrm{Y}$ for C/ T, R for $A / G)$ 


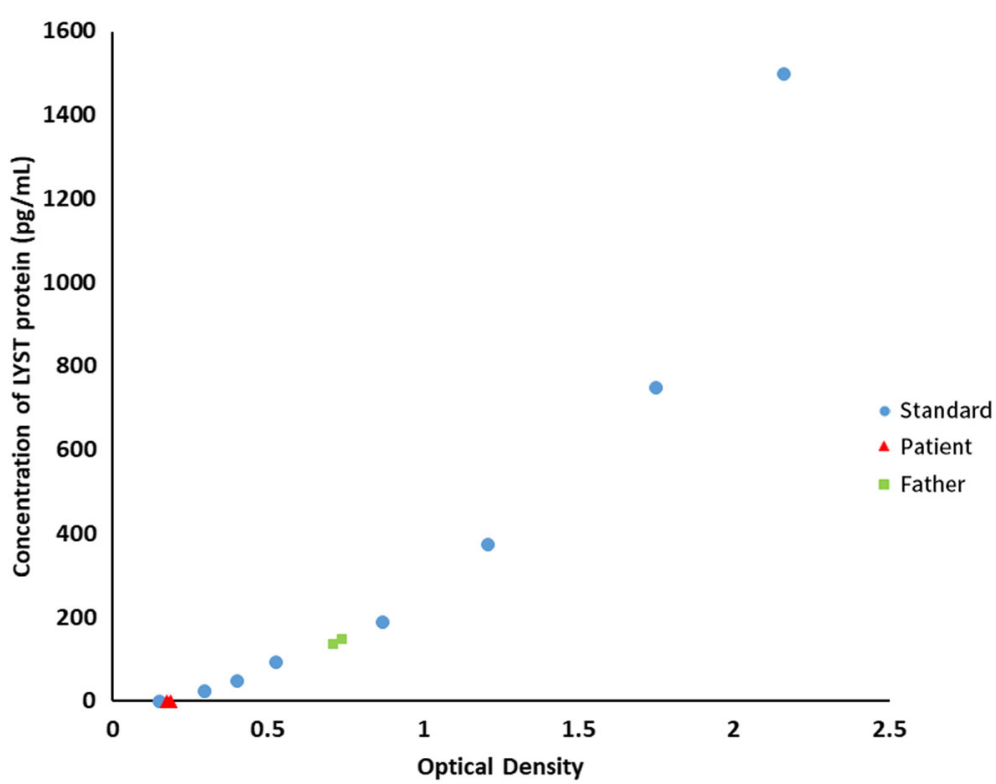

Fig. 3 ELISA test for human LYST protein. Dot: Standards provided in the ELISA kit. Triangle: Plasma sample from the patient. Square: Plasma sample from the patient's father

necessary to monitor the onset of accelerated phase. However, a conflict record had been submitted to ClinVar database by single submitter. According to the $\mathrm{dbSNP}$ and 1000 genomes database, this mutation mainly distributes in Asian people, especially in southwest of China and Southeast Asia. Therefore, further studies are required to confirm the clinical significance of the mutation. On the other hand, a significant disadvantage of exon sequencing should not be neglected, that is the cost-saving method is unable to detect mutations in deep intron. In this report, we couldn't eliminate the possibility that there is other causative mutation locates in deep intron of the LYST gene. A whole-genome sequencing should be performed in further study with the permission of the patient and her family.

Molecular diagnosis for CHS could benefit not only treatment and prognosis, but also differential diagnosis. There are several diseases resemble CHS, including Hermansky Pudlak syndrome 2 (HPS2, MIM: 608233), Griscelli syndrome (GS, OMIM Phenotypic Series: PS214450) and Familial hemophagocytic lymphohistiocytosis (FHL, OMIM Phenotypic Series: PS267700). Amplicon sequencing based on ultrahigh-efficient multiplex PCR provides an approach for detecting mutations from targeted regions on genome. The whole process of DNA preparing, sequencing and data analyzing could be finished in $24 \mathrm{~h}$, which could improve the efficiency in the clinical laboratories.

In summary, a compound heterozygote in LYST gene was identified from a 4-year-old female patient with CHS, a novel missense mutation was included. The result was confirmed by sanger sequencing. The source of mutations was investigated by two-generation pedigree analysis. Our data expands the spectrum of pathogenic mutations in LYST gene, demonstrates the value of amplicon sequencing as an efficient and accurate approach for diagnosis of genetic diseases.

\section{Supplementary information}

Supplementary information accompanies this paper at https://doi.org/10. 1186/s12881-019-0922-8.

Additional file 1: Functional prediction of mutations performed by HSF 3.1, including the novel missense LYST:C.5719A > G, the known SNP rs201382097 (LYST:C.4863-4G > A), and two mutations in adjacent sites of rs201382097, which referred to LYST:C.4863-3 T > A and LYST:C.4863-5 T > A.

Additional file 2: Table S1. Information of custom-designed amplicon sequencing panel for $\mathrm{CHS}$ and related disorders.

Additional file 3: Table S2. Global distribution of the mutation LYST:C.4863-4G>A (rs201382097) in gnomAD database.

Additional file 4: Table S3. Global distribution of the mutation LYST:C.4863-4G>A (rs201382097) in 1000 genome database.

Additional file 5: Table S4. Reported pathogenic and likely pathogenic mutations in the LYST gene from the ClinVar database.

Abbreviations

CHS: Chediak-Higashi Syndrome; HSCT: Hematopoietic stem cell transplantation; PBMC: Peripheral blood mononuclear cells; PLT: Platelets; RBC: Red blood cell; WBC: White blood cell

\section{Acknowledgements}

The authors gratefully appreciate the patient and her family for their participation in this clinical study. This work was supported by the Children's Hospital Affiliated to Zhengzhou University. 


\section{Authors' contributions}

YS, ZD and SL performed the experiments, JY finished the clinical examinations and analyzed the data, YS and YL analyzed sequencing data and wrote the manuscript. BG reviewed and edited the manuscript, TF design this study and edited the manuscript. All authors read and approved the final manuscript.

\section{Funding}

Not applicable.

\section{Availability of data and materials}

The sequence data had been deposited into NCBI Short Read Archive under accession number SRR10382506.

\section{Ethics approval and consent to participate}

The study was approved by the ethics committee of Children's Hospita Affiliated to Zhengzhou University. A written informed consent to participate was obtained from the parents of the patient before performing genetic test.

\section{Consent for publication}

Written informed consent for publishing data and images was obtained from the parents. A copy of the written consent is available for review by the editor of this journal.

\section{Competing interests}

The authors declare that they have no competing interests.

\section{Author details}

${ }^{1}$ Central Laboratory, Zhengzhou People's Hospital Affiliated to Southern Medical University, Children's Hospital Affiliated to Zhengzhou University, Zhengzhou, China. ${ }^{2}$ Binzhou medical University, Yantai, China. ${ }^{3}$ Department of Oncology, Children's Hospital Affiliated to Zhengzhou University, Zhengzhou, China. ${ }^{4}$ Department of Laboratory Medicine, Taihe Hopsital, Hubei University of Medicine, Shiyan, China. ${ }^{5}$ School of Basic Medical Sciences, Zhengzhou University, Zhengzhou 450001, Henan, China.

Received: 4 April 2019 Accepted: 11 November 2019

Published online: 06 January 2020

\section{References}

1. Kaplan J, De Domenico I, Ward DM. Chediak-higashi syndrome. Curr Opin Hematol. 2008;15(1):22-9.

2. Introne W, Boissy RE, Gahl WA. Clinical, molecular, and cell biological aspects of Chediak-Higashi syndrome. Mol Genet Metab. 1999;68(2):283-303.

3. Blume RS, Wolff SM. The Chediak-Higashi syndrome: studies in four patients and a review of the literature. Medicine. 1972;51(4):247-80.

4. Sánchez-Guiu I, Antón Al, García-Barberá N, Navarro-Fernández J, Martínez C, Fuster JL, Couselo JM, Ortuño FJ, Vicente V, Rivera J. Chediak-H igashi syndrome: description of two novel homozygous missense mutations causing divergent clinical phenotype. Eur J Haematol. 2014;92(1):49-58.

5. Certain S, Barrat F, Pastural E, Le Deist F, Goyo-Rivas J, Jabado N, Benkerrou M, Seger R, Vilmer E, Beullier G. Protein truncation test of LYST reveals heterogenous mutations in patients with Chediak-Higashi syndrome. Blood. 2000;95(3):979-83.

6. Higashi O. Congenital gigantism of peroxidase granules. Tohoku J Exp Med. 1954;59(3):315-32

7. Nagle DL, Karim MA, Woolf EA, Holmgren L, Bork P, Misumi DJ, McGrail SH, Dussault BJ Jr, Perou CM, Boissy RE. Identification and mutation analysis of the complete gene for Chediak-Higashi syndrome. Nat Genet. 1996;14(3):307.

8. Dufourcq-Lagelouse R, Lambert N, Duval M, Viot G, Vilmer E, Fischer A, Prieur M, de Saint BG. Chediak-Higashi syndrome associated with maternal uniparental isodisomy of chromosome 1. Eur J Hum Genet. 1999;7(6):633.

9. Karim MA, Suzuki K, Fukai K, Oh J, Nagle DL, Moore KJ, Barbosa E, FalikBorenstein T, Filipovich A, Ishida Y. Apparent genotype-phenotype correlation in childhood, adolescent, and adult Chediak-Higashi syndrome. Am J Med Genet. 2002;108(1):16-22.

10. Faigle W, Raposo G, Tenza D, Pinet V, Vogt AB, Kropshofer H, Fischer A, de Saint-Basile G, Amigorena S. Deficient peptide loading and MHC class II endosomal sorting in a human genetic immunodeficiency disease: the Chediak-Higashi syndrome. J Cell Biol. 1998;141(5):1121-34.
11. Sim N, Kumar P, Hu J, Henikoff S, Schneider G, Ng PC. SIFT web server: predicting effects of amino acid substitutions on proteins. Nucleic Acids Res. 2012;40:452-7.

12. Desmet F, Hamroun D, Lalande M, Collodberoud G, Claustres M, Beroud C Human Splicing Finder: an online bioinformatics tool to predict splicing signals. Nucleic Acids Res. 2009;37(9):e67.

13. Westbroek W, Adams DR, Huizing M, Koshoffer A, Dorward H, Tinloy B, Parkes J, Helipwooley A, Kleta R, Tsilou E. Cellular defects in Chediak-Higashi syndrome correlate with the molecular genotype and clinical phenotype. J Investig Dermatol. 2007;127(11):2674-7.

\section{Publisher's Note}

Springer Nature remains neutral with regard to jurisdictional claims in published maps and institutional affiliations.

\section{Ready to submit your research? Choose BMC and benefit from:}

- fast, convenient online submission

- thorough peer review by experienced researchers in your field

- rapid publication on acceptance

- support for research data, including large and complex data types

- gold Open Access which fosters wider collaboration and increased citations

- maximum visibility for your research: over $100 \mathrm{M}$ website views per year

At BMC, research is always in progress.

Learn more biomedcentral.com/submissions 